International Journal of Pure and Applied Mathematics

Volume 98 No. 2 2015, 169-180

ISSN: 1311-8080 (printed version); ISSN: 1314-3395 (on-line version)

url: http://www.ijpam.eu

doi: http://dx.doi.org/10.12732/ijpam.v98i2.1

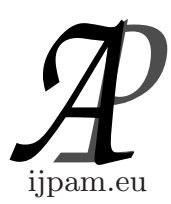

\title{
A GENERALIZATION OF OSTROWSKI TYPE INEQUALITY FOR MAPPINGS WHOSE SECOND DERIVATIVES BELONG TO $\mathrm{L}_{1}(a, b)$ AND APPLICATIONS
}

\author{
A. Qayyum ${ }^{1}$, I. Faye ${ }^{2}$, M. Shoaib ${ }^{3}$, M.A. Latif ${ }^{4}$ \\ ${ }^{1,2}$ Department of Fundamental and Applied Sciences \\ Petronas University of Teknologi \\ Bandar Seri Iskandar, 31750 Tronoh, Perak, MALAYSIA \\ ${ }^{3}$ Department of Mathematics \\ University of Hail \\ P.O. Box 2440, KINGDOM OF SAUDI ARABIA \\ ${ }^{4}$ School of Computational and Applied Mathematics \\ University of the Witwatersrand \\ Private Bag 3, Wits 2050, Johannesburg, SOUTH AFRICA
}

\begin{abstract}
In this paper, we will improve and generalize inequality of Ostrowski type for mappings whose second derivatives belong to $\mathrm{L}_{1}(a, b)$. Some well known inequalities can be derived as special cases. In addition, perturbed mid-point inequality and perturbed trapezoid inequality are also obtained. The obtained inequalities have immediate applications in numerical integration where new estimates are obtained for the remainder term of the trapezoid and midpoint formula. Applications to some special means are also investigated.
\end{abstract}

AMS Subject Classification: 26D15, 41A55, 41A80, 65C50

Key Words: Ostrowski inequality, special means, numerical integration

Received: April 18, 2014

(C) 2015 Academic Publications, Ltd. url: www.acadpubl.eu

${ }^{\S}$ Correspondence author 


\section{Introduction}

The development of inequalities has been established with the publication of the books by Hardy, Littlewood and Polya [1] in 1934, Beckenbach and Bellman [2] in 1961 and by Mitrinovi'c, Pecari'c and Fink [3]-[4] in 1991. The publication of later has resulted to bring forward some new integral inequalities involving functions with bounded derivatives that measure bounds on the deviation of functional value from its mean value namely, Ostrowski inequality [5]. This Ostrowski type inequality has powerful applications in numerical integration, probability and optimization theory, stochastic, statistics, information and integral operator theory.

During the last few years, many researchers focused their attention on the study and generalizations [6], [7], [8], [13], [14], [15], [16] and [17] of the Ostrowski inequality. In [9], Rafiq et.al proved their results for second differentiable mappings by using Ostrowski-Grüss type inequality. We prove our result for $\mathrm{L}_{1}$ norm by using only Ostrowski type inequality.

Ostrowski [5] proved the classical integral inequality which is stated as:

Theorem 1. Let $f: I \subseteq \mathbb{R} \rightarrow \mathbb{R}$ be a differentiable mapping on $I^{\circ}\left(I^{\circ}\right.$ is the interior of $I)$ and let $a, b \in I^{\circ}$ with $a<b$. If $f^{\prime}:(a, b) \rightarrow \mathbb{R}$ is bounded on $(a, b)$ i.e. $\left\|f^{\prime}\right\|_{\infty}=\sup _{t \in[a, b]}\left|f^{\prime \prime}(t)\right|<\infty$, then

$$
\left|f(x)-\frac{1}{b-a} \int_{a}^{b} f(t) d t\right| \leq\left[\frac{1}{4}+\frac{\left(x-\frac{a+b}{2}\right)^{2}}{(b-a)^{2}}\right](b-a)\left\|f^{\prime}\right\|_{\infty},
$$

for all $x \in[a, b]$. The constant $\frac{1}{4}$ is sharp in the sense that it can not be replaced by a smaller one.

In 1976, Milovanović et al. proved a generalization of Ostrowski's inequality for twice differentiable mappings [10].

Theorem 2. Let $f:[a, b] \rightarrow \mathbb{R}$ be a twice differentiable such that $f^{\prime \prime}$ : $(a, b) \rightarrow \mathbb{R}$ is bounded on $(a, b)$, i.e $\left\|f^{\prime \prime}\right\|_{\infty}=\sup _{t \in[a, b]}\left|f^{\prime \prime}(t)\right|<\infty$. Then the inequality holds:

$$
\begin{aligned}
\left|\frac{1}{2}\left[f(x)+\frac{(x-a) f(a)+(b-x) f(b)}{b-a}\right]-\frac{1}{b-a} \int_{a}^{b} f(t) d t\right| \\
\leq \frac{\left\|f^{\prime}\right\|_{\infty}}{4}(b-a)^{2}\left[\frac{1}{12}+\frac{\left(x-\frac{a+b}{2}\right)^{2}}{(b-a)^{2}}\right],
\end{aligned}
$$


for all $x \in[a, b]$.

Dragomir and Wang [7] proved (1.1) for $f^{\prime} \in L_{1}[a, b]$, as follows:

Theorem 3. Let $f: I \subseteq \mathbb{R} \rightarrow \mathbb{R}$ be a differentiable mapping in $I^{\circ}$ and $a, b \in I^{\circ}$ with $a<b$. If $f^{\prime} \in L_{1}[a, b]$, then the inequality holds

$$
\left|f(x)-\frac{1}{b-a} \int_{a}^{b} f(t) d t\right| \leq\left[\frac{1}{2}+\frac{\left|x-\frac{a+b}{2}\right|}{b-a}\right]\left\|f^{\prime}\right\|_{1}
$$

for all $x \in[a, b]$.

Roumeliotis [11], presented product inequalities and weighted quadrature. The weighted inequality was also obtained in Lebesgue spaces involving first derivative of the function, which is given by

$$
\begin{aligned}
\mid \frac{1}{b-a} \int_{a}^{b} w(t) f(t) d t-m & (a, b) f(x) \mid \\
& \leq \frac{1}{2}[m(a, b)+|m(a, x)-m(x, b)|]\left\|f^{\prime \prime}\right\|_{1} .
\end{aligned}
$$

Barnett et,al, [12] proved an inequality of Ostrowski type for twice differentiable mappings which is in terms of the $L_{1}$ norm of the second derivative $f^{\prime \prime}$. The main result from [12] is pointed out in the theorem below:

Theorem 4. Let $f:[a, b] \rightarrow \mathbb{R}$ be continuous on $[a, b]$ and twice differentiable in $(a, b)$ and $f^{\prime \prime} \in L_{1}(a, b)$. Then the inequality holds

$$
\begin{aligned}
& \left|f(x)-\frac{1}{b-a} \int_{a}^{b} f(t) d t-\left(x-\frac{a+b}{2}\right) f^{\prime}(x)\right| \\
& \quad \leq \frac{1}{2(b-a)}\left(\left|x-\frac{a+b}{2}\right|+\frac{1}{2}(b-a)\right)^{2}\left\|f^{\prime \prime}\right\|_{1} \leq \frac{b-a}{2}\left\|f^{\prime \prime}\right\|_{1},
\end{aligned}
$$

for all $x \in[a, b]$.

Motivated and inspired by the work of the Barnett et.al [12], we will establish a new generalized inequality. Some other interesting inequalities are also presented as special cases. In the last, we will present applications for some special means and in numerical integration. 


\section{Main Results}

Theorem 5. Let $f:[a, b] \rightarrow \mathbb{R}$ be continuous on $[a, b]$ and twice differentiable on $(a, b)$ with second derivative and $f^{\prime \prime}:(a, b) \rightarrow \mathbb{R}$, for all $x \in\left[a+h \frac{b-a}{2}, b-h \frac{b-a}{2}\right]$, it follows that

$$
\begin{aligned}
& (1-h) f(x)-(1-h)\left(x-\frac{a+b}{2}\right) f^{\prime}(x)+\frac{h}{2}(f(a)+f(b)) \\
& -\frac{h^{2}(b-a)}{8}\left(f^{\prime}(b)-f^{\prime}(a)\right)-\frac{1}{b-a} \int_{a}^{b} f(t) d t \\
& \leq \frac{1}{2(b-a)}\left[\frac{1}{2}(b-a)(1-h)+\left|x-\frac{a+b}{2}\right|\right]^{2}\left\|f^{\prime \prime}\right\|_{1} \\
& \leq \frac{(b-a)}{2}\left(1-\frac{h}{2}\right)^{2}\left\|f^{\prime \prime}\right\|_{1}
\end{aligned}
$$

for all $x \in\left[a+h \frac{b-a}{2}, b-h \frac{b-a}{2}\right]$ and $h \in[0,1]$.

Proof. Let us define the mapping $K:[a, b]^{2} \rightarrow \mathbb{R}$ by

$$
K(x, t)=\left\{\begin{array}{l}
\frac{1}{2}\left[t-\left(a+h \frac{b-a}{2}\right)\right]^{2}, \text { if } t \in[a, x], \\
\frac{1}{2}\left[t-\left(b-h \frac{b-a}{2}\right)\right]^{2}, \text { if } t \in(x, b] .
\end{array}\right.
$$

The proof uses the following identity:

$$
\begin{aligned}
\int_{a}^{b} f(t) d t & =(b-a)(1-h) f(x)-(b-a)(1-h)\left(x-\frac{a+b}{2}\right) f^{\prime}(x) \\
& +h \frac{b-a}{2}(f(a)+f(b))-\frac{h^{2}(b-a)^{2}}{8}\left(f^{\prime}(b)-f^{\prime}(a)\right) \\
& +\int_{a}^{b} K(x, t) f^{\prime \prime}(t) d t,
\end{aligned}
$$

for all $x \in\left[a+h \frac{b-a}{2}, b-h \frac{b-a}{2}\right]$. Using the identity (2.2), we have

$$
\mid \begin{aligned}
& (1-h) f(x)-\frac{1}{(b-a)} \int_{a}^{b} f(t) d t+\frac{h}{2(1-h)}(f(a)+f(b)) \\
& -\frac{h^{2}(b-a)}{8}\left(f^{\prime}(b)-f^{\prime}(a)\right)-(1-h)\left(x-\frac{a+b}{2}\right) f^{\prime}(x)
\end{aligned}
$$




$$
\begin{aligned}
& =\frac{1}{(b-a)}\left|\int_{a}^{b} K(x, t) f^{\prime \prime}(t) d t\right| \\
& =\frac{1}{(b-a)}\left|\int_{a}^{x} \frac{\left[t-\left(a+h \frac{b-a}{2}\right)\right]^{2}}{2} f^{\prime \prime}(t) d t+\int_{x}^{b} \frac{\left[t-\left(b-h \frac{b-a}{2}\right)\right]^{2}}{2} f^{\prime \prime}(t) d t\right| \\
& \leq \frac{1}{(b-a)}\left[\frac{\left[x-\left(a+h \frac{b-a}{2}\right)\right]^{2}}{2} \int_{a}^{x}\left\|f^{\prime \prime}(t)\right\| d t+\frac{\left[\left(b-h \frac{b-a}{2}\right)-x\right]^{2}}{2} \int_{x}^{b}\left\|f^{\prime \prime}(t)\right\| d t\right. \\
& \leq \frac{1}{(b-a)} \max \left\{\frac{\left[x-\left(a+h \frac{b-a}{2}\right)\right]^{2}}{2}, \frac{\left[\left(b-h \frac{b-a}{2}\right)-x\right]^{2}}{2}\right\} \\
& \times\left[\int_{a}^{x}\left\|f^{\prime \prime}(t)\right\| d t+\int_{x}^{b}\left\|f^{\prime \prime}(t)\right\| d t\right.
\end{aligned}
$$

Now observe that

$$
\begin{aligned}
\max \left\{\frac{\left[x-\left(a+h \frac{b-a}{2}\right)\right]^{2}}{2}, \frac{\left[\left(b-h \frac{b-a}{2}\right)-x\right]^{2}}{2}\right\} & \\
& =\frac{1}{2}\left[\frac{1}{2}(b-a)(1-h)+\left|x-\frac{a+b}{2}\right|\right]^{2}
\end{aligned}
$$

Using (2.4) in (2.3), we get our required result (2.1).

Remark 1. For $h=0$, in (2.1), we obtain Barnett's result (1.4). It shows that Barnett's result (1.4) is our special case.

Remark 2. For $h=1$ in (2.1), we obtain another result:

$$
\begin{aligned}
\mid \frac{1}{2}(f(a)+f(b)) & -\frac{(b-a)}{8}\left(f^{\prime}(b)-f^{\prime}(a)\right)-\frac{1}{b-a} \int_{a}^{b} f(t) d t \mid \\
& \leq \frac{1}{2(b-a)}\left[\left|x-\frac{a+b}{2}\right|\right]^{2}\left\|f^{\prime \prime}\right\|_{1} \leq \frac{(b-a)}{8}\left\|f^{\prime \prime}\right\|_{1} .
\end{aligned}
$$

Hence for different values of $h$, we can obtain a variety of results. 
Corollary 6. If $f$ is as in Theorem 5, then we have the following perturbed midpoint inequality:

$$
\left|\begin{array}{c}
(1-h) f\left(\frac{a+b}{2}\right)+\frac{h}{2}(f(a)+f(b)) \\
-\frac{h^{2}(b-a)}{8}\left(f^{\prime}(b)-f^{\prime}(a)\right)-\frac{1}{b-a} \int_{a}^{b} f(t) d t
\end{array}\right| \leq \frac{1}{8}(b-a)(1-h)^{2}\left\|f^{\prime \prime}\right\|_{1}
$$

and for $h=0$, we have

$$
\left|f\left(\frac{a+b}{2}\right)-\frac{1}{b-a} \int_{a}^{b} f(t) d t\right| \leq \frac{(b-a)}{8}\left\|f^{\prime \prime}\right\|_{1} .
$$

Corollary 7. Let $f$ be as in Theorem 5 , then

$$
\begin{aligned}
& \left|\begin{array}{c}
(1-h) \frac{f(a)+f(b)}{2}-(1-h)(b-a) \frac{f^{\prime}(b)-f^{\prime}(a)}{4}+\frac{h}{2}(f(a)+f(b)) \\
-\frac{h^{2}(b-a)}{8}\left(f^{\prime}(b)-f^{\prime}(a)\right)-\frac{1}{b-a} \int_{a}^{b} f(t) d t
\end{array}\right| \\
& \leq \frac{(b-a)}{2}\left(1-\frac{h}{2}\right)^{2}\left\|f^{\prime \prime}\right\|_{1}
\end{aligned}
$$

holds.

Proof. Put $x=a$ and $x=b$ in (2.1), summing up the obtained inequalities, using the triangle inequality and dividing by 2 , we get the required inequality (2.8).

Corollary 8. Let $f$ be as in Theorem 5, then we have the perturbed trapezoidal inequality:

$$
\begin{aligned}
\left|\frac{f(a)+f(b)}{2}-(b-a) \frac{f^{\prime}(b)-f^{\prime}(a)}{4}-\frac{1}{b-a} \int_{a}^{b} f(t) d t\right| \\
\leq \frac{1}{6}(b-a)^{2}\left\|f^{\prime \prime}\right\|_{\infty} .
\end{aligned}
$$

Proof. Put $h=0$, in (2.8).

Remark 3. The estimation provided by (2.8), is similar to that of the classical trapezoidal inequality. 


\section{Applications in Numerical integration}

Let $I_{n}: a=x_{0}<x_{1}<x_{2}<\ldots<x_{n-1}<x_{n}=b$ be a division of the interval $[a, b], \quad \xi_{i} \in\left[x_{i}+\delta \frac{h_{i}}{2}, x_{i+1}-\delta \frac{h_{i}}{2}\right],(i=0,1, \ldots . ., n-1)$ a sequence of intermediate points and $h_{i}=x_{i+1}-x_{i},(i=0,1, \ldots ., n-1)$. then we have the following quadrature rule:

Theorem 9. Let $f:[a, b] \rightarrow \mathbb{R}$ be a twice differentiable on $(a, b)$ whose second derivative and $f^{\prime \prime}:(a, b) \rightarrow \mathbb{R}$ belongs to $L_{1}(a, b)$, i.e $\left\|f^{\prime \prime}\right\|_{1}:=$ $\int_{a}^{b}\left\|f^{\prime \prime}\right\| d t<\infty$. Then the perturbed Riemann's quadrature formula holds:

$$
\int_{a}^{b} f(t) d t=A\left(f, f^{\prime}, I_{n}, \xi, \delta\right)+R\left(f, f^{\prime}, I_{n}, \xi, \delta\right),
$$

where

$$
\begin{aligned}
& A\left(f, f^{\prime}, I_{n}, \xi, \delta\right) \\
& =(1-\delta) \sum_{i=0}^{n-1} h_{i} f\left(\xi_{i}\right)-(1-\delta) \sum_{i=0}^{n-1} h_{i}\left(\xi_{i}-\frac{x_{i}+x_{i+1}}{2}\right) f^{\prime}\left(\xi_{i}\right) \\
& +\frac{\delta}{2} \sum_{i=0}^{n-1} h_{i}\left(f\left(x_{i}\right)+f\left(x_{i+1}\right)\right)-\frac{\delta^{2}}{8} \sum_{i=0}^{n-1} h_{i}^{2}\left(f^{\prime}\left(x_{i+1}\right)-f^{\prime}\left(x_{i}\right)\right)
\end{aligned}
$$

and the remainder $R\left(f, f^{\prime}, I_{n}, \xi, \delta\right)$ satisfies the estimation:

$$
\begin{aligned}
\left|R\left(f, f^{\prime}, I_{n}, \xi, \delta\right)\right| & \leq \frac{1}{2} \sum_{i=0}^{n-1}\left[\frac{h_{i}(1-\delta)}{2}+\left|\xi_{i}-\frac{x_{i}+x_{i+1}}{2}\right|\right]^{2}\left\|f^{\prime \prime}\right\|_{1} \\
& \leq\left(1-\frac{\delta}{2}\right)^{2} \sum_{i=0}^{n-1} \frac{h_{i}^{2}}{2}\left\|f^{\prime \prime}\right\|_{1}
\end{aligned}
$$

where $\delta \in[0,1]$ and $x_{i}+\delta \frac{h_{i}}{2} \leq \xi_{i} \leq x_{i+1}-\delta \frac{h_{i}}{2}$.

Proof. Apply Theorem 5 on the interval $\left[x_{i}, x_{i+1}\right],(i=0,1, \ldots . n-1)$, gives

$$
\begin{gathered}
(1-\delta) h_{i} f\left(\xi_{i}\right)-(1-\delta) h_{i}\left(\xi_{i}-\frac{x_{i}+x_{i+1}}{2}\right) f^{\prime}\left(\xi_{i}\right)+\frac{\delta}{2} h_{i}\left(f\left(x_{i}\right)+f\left(x_{i+1}\right)\right) \\
-\frac{\delta^{2}}{8} h_{i}^{2}\left(f^{\prime}\left(x_{i+1}\right)-f^{\prime}\left(x_{i}\right)\right)-\int_{x_{i}}^{x_{i+1}} f(t) d t
\end{gathered}
$$




$$
\begin{aligned}
& \leq \frac{1}{2}\left[\frac{h_{i}(1-\delta)}{2}+\left|\xi_{i}-\frac{x_{i}+x_{i+1}}{2}\right|\right]^{2}\left\|f^{\prime \prime}\right\|_{1} \\
& \leq\left(1-\frac{\delta}{2}\right)^{2} \frac{h_{i}^{2}}{2}\left\|f^{\prime \prime}\right\|_{1}
\end{aligned}
$$

for any choice $\xi$ of the intermediate points. Summing over $i$ from 0 to $n-1$ and using the generalized triangular inequality, we deduce the desired estimation $(? ?)$.

Corollary 10. The following perturbed midpoint rule holds:

$$
\int_{a}^{b} f(x) d x=M\left(f, f^{\prime}, I_{n}\right)+R_{M}\left(f, f^{\prime}, I_{n}\right)
$$

where

$$
M\left(f, f^{\prime}, I_{n}\right)=\sum_{i=0}^{n-1} h_{i} f\left(\frac{x_{i}+x_{i+1}}{2}\right)
$$

and the remainder term $R_{M}\left(f, f^{\prime}, I_{n}\right)$ satisfies the estimation:

$$
\left|R_{M}\left(f, f^{\prime}, I_{n}\right)\right| \leq\left\|f^{\prime \prime}\right\|_{1} \sum_{i=0}^{n-1} \frac{h_{i}^{2}}{8} .
$$

Corollary 11. The following perturbed trapezoidal rule holds:

$$
\int_{a}^{b} f(x) d x=T\left(f, f^{\prime}, I_{n}\right)+R_{T}\left(f, f^{\prime}, I_{n}\right)
$$

where

$$
T\left(f, f^{\prime}, I_{n}\right)=\frac{1}{2} \sum_{i=0}^{n-1} h_{i}\left(f\left(x_{i}\right)+f\left(x_{i+1}\right)\right)-\frac{1}{8} \sum_{i=0}^{n-1} h_{i}^{2}\left(f^{\prime}\left(x_{i+1}\right)-f^{\prime}\left(x_{i}\right)\right)
$$

and the remainder term

$$
\left|R_{T}\left(f, f^{\prime}, I_{n}\right)\right| \leq \sum_{i=0}^{n-1} \frac{h_{i}^{2}}{8}\left\|f^{\prime \prime}\right\|_{1} .
$$

Remark 4. Note that the above mentioned perturbed midpoint formula (3.4) and perturbed trapezoid formula (3.7) can give better approximations of the integral $\int_{a}^{b} f(x) d x$ for general classes of mappings. 


\section{Special Means}

We may now apply inequality (2.1), to deduce some inequalities for special means [15] at [P. 1896] by the use of particular mappings as follows:

Remark 5. Consider mapping $f:(0, \infty) \rightarrow \mathbb{R}, f(x)=x^{r}, r \in \mathbb{R} \backslash\{-1,0\}$, then we have for $0<a<b$,

$$
\frac{1}{b-a} \int_{a}^{b} f(t) d t=L_{r}^{r}(a, b)
$$

Using the inequality (2.1) we get:

$$
\begin{aligned}
& \left|\begin{array}{c}
(1-h) x^{r}-(1-h)(x-A) r x^{r-1} \\
+\frac{h}{2}\left(a^{r}+b^{r}\right)-\frac{h^{2}(b-a) r}{8}\left[b^{r-1}-a^{r-1}\right]-L_{r}^{r}(a, b)
\end{array}\right| \\
& \leq \frac{1}{2}\left[\frac{1}{2}(b-a)(1-h)+|x-A|\right]^{2}\left|r(r-1) L_{r-1}^{r-1}(a, b)\right| .
\end{aligned}
$$

If in (4.1), we choose $x=A$, we get

$$
\begin{aligned}
& \left|(1-h) A^{r}+\frac{h}{2}\left(a^{r}+b^{r}\right)-\frac{h^{2}(b-a) r}{8}\left[b^{r-1}-a^{r-1}\right]-L_{r}^{r}(a, b)\right| \\
& \leq \frac{1}{8}[(b-a)(1-h)]^{2}\left|r(r-1) L_{r-1}^{r-1}(a, b)\right|
\end{aligned}
$$

also choosing $h=0$ in (4.2), we get

$$
\left|A^{r}-L_{r}^{r}(a, b)\right| \leq \frac{1}{8}(b-a)^{2}\left|r(r-1) L_{r-1}^{r-1}(a, b)\right| .
$$

Remark 6. Consider the mapping $f:(0, \infty) \rightarrow \mathbb{R}, \quad f(x)=\frac{1}{x}$.

Then we have for $0<a<b$,

$$
\frac{1}{b-a} \int_{a}^{b} f(t) d t=L^{-1}(a, b)
$$

Using the inequality (2.1) we get:

$$
\left|(1-h) \frac{1}{x}+(1-h)(x-A) \frac{1}{x^{2}}+\frac{h}{H}-\frac{h^{2}(b-a)}{8}\left(\frac{b^{2}-a^{2}}{a^{2} b^{2}}\right)-L^{-1}(a, b)\right|
$$




$$
\leq\left[\frac{1}{2}(b-a)(1-h)+|x-A|\right]^{2} L_{-3}^{-3}(a, b) .
$$

If in (4.4), we choose $x=A$, we get

$$
\begin{aligned}
& \left|(1-h) \frac{1}{A}+\frac{h}{H}-\frac{h^{2}(b-a)}{8}\left(\frac{b^{2}-a^{2}}{a^{2} b^{2}}\right)-L^{-1}(a, b)\right| \\
& \leq \frac{1}{4}(b-a)^{2}(1-h)^{2} L_{-3}^{-3}(a, b)
\end{aligned}
$$

also choosing $h=0$ in (4.5), we get

$$
\left|A^{-1}-L^{-1}(a, b)\right| \leq \frac{1}{4}(b-a)^{2} L_{-3}^{-3}(a, b) .
$$

Remark 7. Let us consider the mapping $f(x)=\ln x, x \in[a, b] \subset(0, \infty)$. Then we have:

$$
\frac{1}{b-a} \int_{a}^{b} f(t) d t=\ln I(a, b)
$$

Using the inequality (2.1) we get:

$$
\begin{gathered}
\mid \begin{array}{c}
(1-h) \ln x-(1-h)(x-A) \frac{1}{x}+\frac{h}{2}(\ln a+\ln b) \\
+\frac{h^{2}(b-a)^{2}}{8 a b}-\ln I(a, b)
\end{array} \\
\leq \frac{1}{2}\left[\frac{1}{2}(b-a)(1-h)+|x-A|\right]^{2} L_{-2}^{-2}(a, b)
\end{gathered}
$$

If in (4.7), we choose $x=A$, we get

$$
\begin{aligned}
& \left|(1-h) \ln A-\frac{h}{2}(\ln a+\ln b)+\frac{h^{2}(b-a)^{2}}{8 a b}-\ln I(a, b)\right| \\
& \leq \frac{1}{8}(b-a)^{2}(1-h)^{2} L_{-2}^{-2}(a, b)
\end{aligned}
$$

also choosing $h=0$ in (4.8), we get

$$
\left|\frac{A}{I}\right| \leq \exp \frac{(b-a)^{2}}{8} L_{-2}^{-2}(a, b) .
$$




\section{Conclusion}

We established generalized Ostrowski type inequality for bounded differentiable mappings which generalizes the previous inequalities developed and discussed in $[6],[7],[10]-[12]$. Perturbed midpoint and trapezoid inequalities are obtained. Some closely new results are also given. These generalized inequalities add up to the literature in the sense that they have immediate applications in $\mathrm{Nu}-$ merical Integration and Special Means. These generalized inequalities will also be useful for the researchers working in the field of the approximation theory, applied mathematics, probability theory, stochastic and numerical analysis to solve their problems in engineering and in practical life.

\section{References}

[1] G. H. Hardy, J. E. Littlewood and G. Polya, Inequalities, Cambridge University Press, 1934.

[2] E. F. Beckenbach and R. Bellman, Springer-Verlag, Berlin-GottinggonHeidelberg, 1961.

[3] D. S. Mitrinovi 'c , J. E. Pecari'c , and A. M. Fink. "Classical and New Inequalities in Analysis". Kluwer Academic Publishers, Dordrecht (1991).

[4] D. S. Mitrinovi 'c , J. E. Pecari 'c, and A. M. Fink. "Inequalities Involving Functions and their Integrals and Derivatives". Kluwer Academic Publishers, Dordrecht (1991).

[5] A. Ostrowski, Uber die Absolutabweichung einer differentien baren Funktionen von ihren Integralimittelwert, Comment. Math. Hel. 10(1938), 226227.

[6] S.S. Dragomir and S. Wang, A new inequality of Ostrowski's type in $\mathrm{L}_{1}$-norm and applications to some special means and to some numerical quadrature rules, Tamkang J. of Math., 28 (1997), 239-244.

[7] S.S. Dragomir and S. Wang, Applications of Ostrowski's inequality to the estimation of error bounds for some special means and some numerical quadrature rules, Appl. Math. Lett., 11 (1998), 105-109.

[8] S.S. Dragomir, P. Cerone and J. Roumeliotis, A new generalization of Ostrowski integral inequality for mappings whose derivatives are bounded 
and applications in numerical integration and for special means, Appl. Math. Lett., 13 (2000), 19-25.

[9] A. Rafiq, N.A. Mir and Fiza Zafar, A Generalized Ostrowski-Grüss Type Inequality for Twice Differentiable Mappings and Applications, J. Ineq. Pure and Appl. Math. 7(4) Art. 124, 2006.

[10] D. S. Mitrinovi'c , J. E. Pecari'c, and A. M. Fink, Inequalities for Functions and Their Integrals and Derivatives. Kluwer Academic, Dordrecht, 1994.

[11] J. Roumeliotis, Improved weighted Ostrowski Grüss type inequalities. Inequality Theory and Applications (Eds. Y. J. Cho, Y. K. Kim and S.S. Dragomir) Vol. 3, 153-160, Nova Sci. Publ., Hauppauge, NY, 2003.

[12] N.S. Barnett, P. Cerone, S.S. Dragomir, J. Roumeliotis, A. Sofo, A survey on Ostrowski type inequalities for twice differentiable mappings and applications, Inequality Theory and Applications 1 (2001) 24-30.

[13] A. Qayyum and S. Hussain, A new generalized Ostrowski Grüss type inequality and applications, Applied Mathematics Letters 25 (2012) 18751880.

[14] A. Qayyum, M. Shoaib, A. E. Matouk, and M. A. Latif, On New Generalized Ostrowski Type Integral Inequalities, Abstract and Applied Analysis, Volume 2014, Article ID 275806.

[15] A. Qayyum, M. Shoaib, and M. A. Latif, A generalized inequality of ostrowski type for twice differentiable bounded mappings and applications, Applied Mathematical Sciences, Vol. 8, 2014, no. 38, 1889-1901.

[16] A. Qayyum, I. Faye and M. Shoaib, Improvement of Ostrowski Integral Type inequalities with application, Filomat (Accepted).

[17] A. Qayyum, M. Shoaib, and I. Faye, Some New Generalized Results on Ostrowski Type Integral Inequalities With Application, journal of computational analysis and applications, (Accepted). 